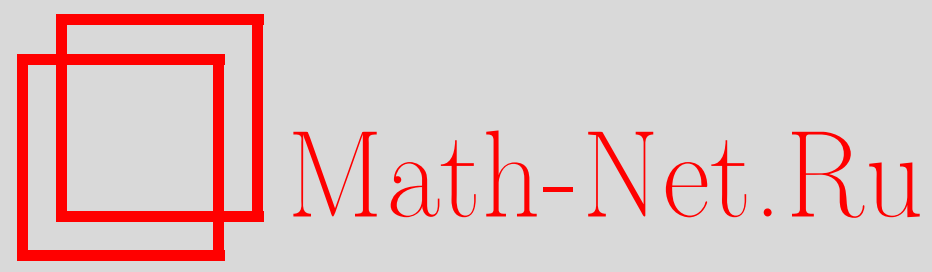

Л. В. Тайков, Одностороннее приближение функций, $M a$ тем. заметки, 2000, том 67, выпуск 1, 136-140

DOI: https://doi.org/10.4213/mzm821

Использование Общероссийского математического портала Math-Net.Ru подразумевает, что вы прочитали и согласны с пользовательским соглашением http://www . mathnet.ru/rus/agreement

Параметры загрузки:

IP : 107.22 .136 .117

26 апреля 2023 г., 17:22:09

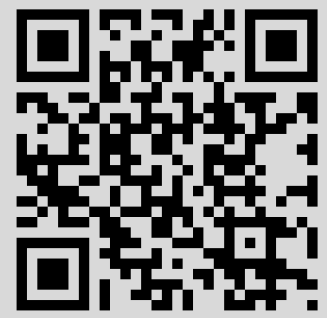




\section{ОДНОСТОРОННЕЕ ПРИБЛИЖЕНИЕ ФУНКЦИЙ}

\section{Л.В. Тайков}

Получено наилучшее одностороннее приближение периодических функций тригонометрическими полиномами класса $W_{p}^{0}(K)$ в метрике $L$.

Библиография: 1 название.

Пусть $\varphi(x)$ - произвольная измеримая $2 \pi$-периодическая функция с нормой пространства $L_{p}[0,2 \pi)$

$$
\|\varphi\|_{p}=\left\{\int_{0}^{2 \pi}\left|\varphi_{x}\right|^{p}\right\}^{\frac{1}{p}}=1,
$$

где $p$ - любое заданное число, $1<p<\infty$. Если $K(x) \in L_{q}[0,2 \pi), 1 / q+1 / p=1$, то определим класс непрерьвных функций $\{f(x)\}=W_{p}(K) \subset C[0,2 \pi)$, представимых сверткой

$$
f(x)=c+\frac{1}{\pi} \int_{0}^{2 \pi} K(x-t) \cdot \varphi(t) d t=C+K * \varphi .
$$

Будем допускать также случай: $p=\infty, q=1$, рассматривая $\|\varphi\|_{\infty}$ как существенньй максимум, и если $p=1, q=\infty$, то ограничимся только непрерьвньми $K(x)$, $K(x) \in C[0,2 \pi)$, и $\varphi(t) d t=d v(t)$, где $v(t)$ - функция ограниченной вариации. Смысл этих ограничений состоит в том, чтобы пространство $L_{p}[0,2 \pi)$ всегда было сопряженным пространством, т.е. пространством функционалов для некоторого банахового пространства. В этом смысле показатель $p$ допускает ограничения $1 \leqslant p \leqslant \infty$.

Если дополнительно все функции $\varphi(x)$ ортогональны 1 :

$$
\int_{0}^{2 \pi} \varphi(x) d x=0, \quad 1<p \leqslant \infty ; \quad \int_{0}^{2 \pi} d v(t)=0, \quad p=1
$$

то такой класс функций $\{f(x)\}$ будем обозначать $W_{p}^{0}(K)$.

Наилучшее приближение $K(x)$ тригонометрическими полиномами

$$
T_{n-1}(x)=\frac{a_{0}}{2}+\sum_{k=1}^{n-1}\left(a_{k} \cos k x+b_{k} \sin k x\right)
$$

определим как обычно:

$$
\inf _{T_{n-1}}\left\|K-T_{n-1}\right\|_{q}=E_{n}(K)_{q}
$$


наилучшее приближение сверху функции $f(x)$ - как

$$
\inf _{T_{n-1} \geqslant f}\left\|f-T_{n-1}\right\|_{1}=E_{n}^{+}(f)_{1} .
$$

Аналогично определяется наилучшее приближение снизу

$$
\inf _{T_{n-1} \leqslant f}\left\|f-T_{n-1}\right\|_{1}=E_{n}^{-}(f)_{1} .
$$

И, наконец, определим соответствующие односторонние приближения классов функции как

$$
\sup _{f \in W_{p}(K)} E_{n}^{ \pm}(f)_{1}=E_{n}^{ \pm}\left\{W_{p}(K)\right\}_{1} .
$$

Мы привели в соответствие с монографией [1] данные вьше определения и обозначения и теперь в состоянии процитировать следующий результат:

Tеорема 4.2.2. Для всех $n=1,2, \ldots$

$$
E_{n}^{+}\left\{W_{\infty}^{0}(K)\right\}_{1} \leqslant 2 E_{n}(K)_{1},
$$

при этом если $E_{n}(K)_{1}=\left|\int_{0}^{2 \pi} K(t) \operatorname{sign} \sin n\left(t-\gamma_{0}\right) d t\right|$, то предыдущее неравенство обращается в равенство.

В настоящей работе предполагается уточнить теорему 4.2.2 и в этой уточненной форме распространить ее на классы $W_{p}^{0}(K)$.

Теорема 1. Для любого натурального $n$ и любого $p, 1 \leqslant p \leqslant \infty$, справедливо равенство

$$
E_{n}^{+}\left\{W_{p}^{0}(K)\right\}_{1}=2 E_{n}(K)_{q} .
$$

Следовательно, теорема 1 при $p=\infty, q=1$ уточняет теорему 4.2.2.

ДоКАЗАТЕЛЬСТво ТЕОРЕмЫ 1. Оно по основньм пунктам следует доказательству теоремы 4.2.2 с очевидными изменениями в соответствуюших местах.

1. Оценка сверху. Пусть $f \in W_{p}(K)$ и, следовательно, представима в виде $f(x)=$ $C+K * \varphi$. Если $T_{n-1}(K)$ полином наилучшего приближения $\left\|K-T_{n-1}(K)\right\|_{q}=$ $E_{n}(K)_{q}$, то разность

$$
f(x)-T_{n-1}(f, x)=\left(K-T_{n-1}(K)\right) * \varphi,
$$

где $T_{n-1}(f, x)=C+T_{n-1}(K) * \varphi$ будет ортогональна константе. С другой стороны, мы можем оценить

$$
\left\|f-T_{n-1}(f)\right\|_{\infty}=\left\|\left(K+T_{n-1}(K)\right) * \varphi\right\|_{\infty} \leqslant \frac{1}{\pi}\left\|K-T_{n-1}(K)\right\|_{q} \cdot\|\varphi\|_{p} \leqslant \frac{1}{\pi} E_{n}(K)_{q}
$$

и, следовательно, для любого $x \in[0,2 \pi)$

$$
T_{n-1}(f, x)-\frac{1}{\pi} E_{n}(K)_{q} \leqslant f(x) \leqslant T_{n-1}(f, x)+\frac{1}{\pi} E_{n}(K)_{q} .
$$

Тригонометрический полином

$$
T_{n-1}^{+}(f, x)=T_{n-1}(f, x)+\frac{1}{\pi} E_{n}(K)_{q}
$$

расположен над функцией $f(x)$, и поэтому

$E_{n}^{+}(f)_{1} \leqslant\left\|T_{n-1}^{+}(f, x)-f(x)\right\|_{1}=\int_{0}^{2 \pi}\left\{T_{n-1}(f, x)-f(x)+\frac{1}{\pi} E_{n}(K)_{q}\right\} d x=2 E_{n}(K)_{q} ;$ здесь мы воспользовались тем, что интеграл от разности полинома и функции равен нулю. 
2. Оценка снизу. Рассмотрим функцию

$$
\varphi^{*}(t)=\operatorname{sign}\left\{K(t)-T_{n-1}(K, t)\right\} \cdot \frac{\left|K(t)-T_{n-1}(K, t)\right|^{q-1}}{\left\|\left|K-T_{n-1}(K)\right|^{q-1}\right\|_{p}} ;
$$

поскольку $T_{n-1}(K, t)$ является полиномом наилучшего приближения, $\varphi^{*}(t)$ обладает следующими свойствами: она ортогональна любому полиному $T_{n-1}(t),\left\|\varphi^{*}\right\|_{p}=1$,

$$
E_{n}(K)_{q}=\int_{0}^{2 \pi} K(t) \cdot \varphi^{*}(t) d t
$$

В связи с последним свойством свертка вспомогательной функции $\varphi_{1}(t)=\varphi^{*}(-t)$ с $K(t)$ имеет максимальное значение

$$
\frac{1}{\pi} E_{n}(K)_{q}: \max _{x} K * \varphi_{1}=\frac{1}{\pi} E_{n}(K)_{q} .
$$

Положим $f_{1}(x)=K * \varphi_{1}$ и рассмотрим $f_{2}(x)=f_{1}(n x)$. Эта функция из класса $W_{p}^{0}(K)$ и $2 \pi / n$ периодична; тогда на основании леммы 3.1 .2 из [1]

$$
E_{n}^{+}\left(f_{2}\right)_{1}=\int_{0}^{2 \pi}\left\{\max _{x} f_{2}(x)-f_{2}(t)\right\} d t=\int_{0}^{2 \pi} \max _{x} f_{1}(x) d t=2 \pi \cdot \frac{1}{\pi} \cdot E_{n}(K)_{q}
$$

и оценка снизу доказана.

Для дальнейшего сделаем следующее замечание. Пусть задано произвольное непрерьвное ядро $K(x)$ и $v(x)$ - произвольная функция ограниченной вариации. Свертка $f(x)=K * d v$ принадлежит классу $W_{1}(K)$; тогда существует последовательность суммируемых функций $\left\{\varphi_{n}(x)\right\}$ такая, что в смысле равномерной сходимости

$$
f_{n}(x)=K * \varphi_{n}, \quad f_{n}(x) \rightrightarrows f(x) .
$$

Это следует из того, что $\delta$ - функция в смысле слабой сходимости аппроксимируется некоторой последовательностью суммируемых функций, и если вариация $V(v)=1$ и $d v(x) \perp$ $C$, то можно найти последовательность суммируемых функций $\psi_{n}(x),\left\|\psi_{n}\right\|_{1}=1$, $\psi_{n} \perp C$, которая слабо сходится к $d v(x)$.

В монографии [1] класс $W_{1}^{0}(K)$ понимается как совокупность $\{f(x)\}$ функций, представимых сверткой

$$
f(x)=C+K * \varphi, \quad\|\varphi\|_{1} \leqslant 1, \quad \varphi(x) \perp C .
$$

Для любого непрерывного ядра $K(x)$ он образует всюду плотное множество в классе функций $f(x)=C+K * d v, V(v) \leqslant 1, d v \perp C$; следовательно, величина $E_{n}^{+}\left\{W_{1}^{0}(K)\right\}_{1}$ в обоих случаях одна и та же.

Из теоремы 4.2.3 монографии [1] с любым непрерывным ядром $K(x)$ следует оценка

$$
E_{n}^{+}\left\{W_{1}^{0}(K)\right\}_{1} \leqslant \frac{1}{2 \pi}\left\{E_{n}^{+}(K)_{1}+E_{n}^{-}(K)_{1}\right\}
$$

Поставим задачу о построении полиномов наилучших односторонних приближений в $L_{1}[0,2 \pi) T_{n-1}^{+}(K, x)$ и $T_{n-1}^{-}(K, x)$ для любой непрерывной функции.

Пусть $T_{n-1}(K, x)$ - полином наилучшего приближения $K(x)$ в метрике $C[0,2 \pi]$, $\left\|K-T_{n-1}(K)\right\|_{\infty}=E_{n}(K)_{\infty}$. 
ТЕОРемА 2. Для любой заданной непрерывной функиии $K(x)$ ее полиномом наилучшего приближения сверху в метрике пространства $L_{1}[0,2 \pi)$ является

$$
T_{n-1}^{+}(K, x)=E_{n}(K)_{\infty}+T_{n-1}(K, x)
$$

и соответственно полиномом снизу

$$
T_{n-1}^{-}(K, x)=-E_{n}(K)_{\infty}+T_{n-1}(K, x) .
$$

Таким образом,

$$
\begin{aligned}
& E_{n}^{+}(K)_{1}=\int_{0}^{2 \pi}\left(E_{n}(K)_{\infty}+T_{n-1}(K, x)-K(x)\right) d x, \\
& E_{n}^{-}(K)_{1}=\int_{0}^{2 \pi}\left(K(x)+E_{n}(K)_{\infty}-T_{n-1}(K, x)\right) d x .
\end{aligned}
$$

ДокАЗАТЕЛЬСТво. Временно переобозначим введенные в теореме 2 полиномы:

$$
\begin{aligned}
& t_{n-1}^{+}(K, x)=E_{n}(K)_{\infty}+T_{n-1}(K, x), \\
& t_{n-1}^{-}(K, x)=-E_{n}(K)_{\infty}+T_{n-1}(K, x) .
\end{aligned}
$$

Поскольку $\left\|K-T_{n-1}(K)\right\|_{\infty}=E_{n}(K)_{\infty}$, для любого $x \in[0,2 \pi)$

$$
t_{n-1}^{-}(K)=-E_{n}(K)+T_{n-1}(K, x) \leqslant K(x) \leqslant E_{n}(K)_{\infty}+T_{n-1}(K, x)=t_{n-1}^{+}(K, x) .
$$

Кстати, оба полинома $t_{n-1}^{+}(K, x)$ и $t_{n-1}^{-}(K, x)$ удовлетворяют необходимым условиям полинома наилучшего одностороннего приближения.

Временно обозначим также

$$
\begin{aligned}
& e_{n}^{+}(K)_{1}=\int_{0}^{2 \pi}\left\{t_{n-1}^{+}(K, x)-K(x)\right\} d x, \\
& e_{n}^{-}(K)_{1}=\int_{0}^{2 \pi}\left\{K(x)-t_{n-1}^{-}(K, x)\right\} d x .
\end{aligned}
$$

На основании оценки (1) и теоремы 1 имеем

$$
2 E_{n}(K)_{\infty}=E_{n}^{+}\left\{W_{1}^{0}(K)\right\}_{1} \leqslant \frac{1}{2 \pi}\left\{E_{n}^{+}(K)_{1}+E_{n}^{-}(K)_{1}\right\} .
$$

Отсюда следует

$$
4 \pi E_{n}(K)_{\infty} \leqslant E_{n}^{+}(K)_{1}+E_{n}^{-}(K)_{1} .
$$

Величина слева есть площадь прямоугольника, расположенного между

$$
y=-E_{n}(K)_{\infty} \text { и } y=E_{n}(K)_{\infty}, \quad 0 \leqslant x \leqslant 2 \pi .
$$

Он разбит графиком функции $y=-K(x)+T_{n-1}(K, x)$ на две части. Площадь под графиком этой функции равна $e_{n}^{+}(K)_{1}$, а площадь над графиком равна $e_{n}^{-}(K)_{1}$. Следовательно,

$$
e_{n}^{+}(K)_{1}+e_{n}^{-}(K)_{1} \leqslant E_{n}^{+}(K)_{1}+E_{n}^{-}(K)_{1} .
$$

Отсюда сначала следует

$$
e_{n}^{+}(K)_{1}+e_{n}^{-}(K)_{1}=E_{n}^{+}(K)_{1}+E_{n}^{-}(K)_{1},
$$

а затем $e_{n}^{+}(K)_{1}=E_{n}^{+}(K)_{1}, e_{n}^{-}(K)_{1}=E_{n}^{-}(K)_{1}$ и теорема 2 полностью доказана. 
СлЕДСТвИЕ. Для любой непрерывной функиии $K(x)$ справедливо равенство

$$
4 \pi E_{n}(K)_{\infty}=E_{n}^{+}(K)_{1}+E_{n}^{-}(K)_{1} .
$$

В частности, для ядра Пуассона $P_{\rho}(x)$ имеем

$$
4 \pi E_{n}\left(P_{\rho}\right)_{\infty}=2 \pi \cdot \frac{\rho^{n}}{1-\rho^{n}}+2 \pi \cdot \frac{\rho^{n}}{1+\rho^{n}} ; \quad E_{n}\left(P_{\rho}\right)_{\infty}=\frac{\rho^{n}}{1-\rho^{2 n}} .
$$

Такое же выражение имеет $E_{n}\left(\widetilde{P}_{\rho}\right)_{\infty}$ для сопряженного ядра Пуассона $\widetilde{P}_{\rho}(x)$.

3. Односторонний поперечник. Известно, что поперечник

$$
d_{2 n-1}(K)=\inf _{L_{2 n-1}} \sup _{\varphi \in K} \inf _{f \in L_{2 n-1}}\|\varphi-f\|
$$

шара большей размерности равен радиусу шара $K=\left\{u:\|u\| \leqslant r, u \in L_{2 n+1}\right\}, r$. Пусть

$$
K=U_{2 n+1}=\left\{u:\|u\| \leqslant r, u \in L_{2 n+1}\right\}, \quad \operatorname{dim} U_{2 n+1}=2 n+1,
$$

и тогда $d_{2 n-1}\left(U_{2 n+1}\right)=r$. Конечномерность произвольного подпространства $L_{2 n-1}$ обеспечивает существование элементов $u \in U_{2 n+1}$ и $f \in L_{2 n-1}$, для которых $d_{2 n-1}(K)=\|u-f\|$, при этом гиперплоскость $L_{2 n-1}+u$ реализует одностороннее приближение $U_{2 n+1}$, и существуют элементы $u^{*} \in U_{2 n+1}, f^{*} \in L_{2 n-1}+u^{*}$, на которых достигаются

$$
\sup _{u \in U_{2 n+1}} \inf _{f \in L_{2 n-1}+u^{*}}\|u-f\|=\left\|u^{*}-f^{*}\right\|
$$

и одностороннееприближение шара $U_{2 n+1}$ всеми элементами, которые лежат по другую сторону гиперплоскости $L_{2 n-1}+u^{*}$ от шара $U_{2 n+1}$. Таким образом, односторонний поперечник размерности $2 n-1$

$$
d_{2 n-1}\left(U_{2 n+1}\right)=\inf _{\substack{L_{2 n-1+u} \\\|u\| \geqslant r}} \sup _{\varphi \in U_{2 n+1}} \inf _{v \in L_{2 n-1}+u}\|\varphi-v\|
$$

$(2 n+1)$-мерного шара $U_{2 n+1}$ совпадает с $2 r$.

\section{СПИСОК ЦИТИРОВАННОЙ ЛИТЕРАТУРЫ}

[1] Корнейчук Н. П., Лигун А. А., Доронин В. Г. Аппроксимация с ограничениями. Киев: Наукова думка, 1982. 